\title{
Remitting Seronegative Symmetrical Synovitis with Pitting Oedema as the First Manifestation of an Adenocarcinoma of the Caecum
}

\author{
Edgar Pratas $^{1,2}$, João Carvalho ${ }^{1,2}$, Isabel Domingues ${ }^{1,2}$, João Fonseca ${ }^{1}$, Teresa Carvalho $^{2}$, \\ Gabriela Sousa², Manuel Teixeira Veríssimo ${ }^{1}$ \\ ${ }^{1}$ Internal Medicine Department, Centro Hospitalar e Universitário de Coimbra, Coimbra, Portugal \\ ${ }^{2}$ Medical Oncology Department, Instituto Português Oncologia de Coimbra Francisco Gentil E. P. E., Coimbra, Portugal
}

Received: 01/11/2018

Accepted: 09/11/2018

Published: $22 / 11 / 2018$

\begin{abstract}
How to cite this article: Pratas E, Carvalho J, Domingues I, Fonseca J, Carvalho T, Sousa G. Texeira Veríssimo M. Remitting seronegative symmetrical synovitis with pitting oedema as the first manifestation of an adenocarcinoma of the caecum. EJCRIM 2018;5: doi:10.12890/2018_000976.
\end{abstract}

Conflicts of Interests: The Authors declare that there are no competing interests.

This article is licensed under a Commons Attribution Non-Commercial 4.0 License

\section{ABSTRACT}

Remitting seronegative symmetrical synovitis with pitting oedema (RS3PE) is a rare syndrome that affects the elderly. Although the aetiology is not fully understood, it has been related to multiple diseases including cancer. We present the case of an 80-year-old man with a full spectrum of signs and symptoms compatible with RS3PE: sudden onset of bilateral polyarthralgia of the metacarpophalangeal and proximal interphalangeal joints, oedema of the dorsum of the hands, increased inflammatory markers and seronegative rheumatoid factor. After a 5-day course of corticotherapy, the patient became asymptomatic although maintaining a mild anaemia. During a search for the underlying cause of the RS3PE, an early stage adenocarcinoma of the caecum was diagnosed and surgically removed. No further treatment was performed and after 3 years of follow-up the patient remains without evidence of either paraneoplastic RS3PE or cancer.

\section{LEARNING POINTS}

- RS3PE3 is a rare inflammatory syndrome characterized by symmetrical distal synovitis and pitting oedema over the dorsum of the hands and/or feet and seronegative rheumatoid factor.

- The pathogenesis has not been fully explained although it has been described with multiple diseases including as a paraneoplastic syndrome.

- Our clinical awareness led to prompt diagnosis of an early stage and curable adenocarcinoma of the caecum.

\section{KEYWORDS}

RS3PE, paraneoplastic, adenocarcinoma, cecum

\section{CASE PRESENTATION}

In August 2015 an 80-year-old man, previously Eastern Cooperative Oncology Group Performance Status 0, was admitted to our institution with acute onset of bilateral polyarthralgia of the metacarpophalangeal and proximal interphalangeal joints as well as oedema of the dorsum of the hands. He rated the continuous pain as 6/10 (on a numeric scale) and pain exacerbations with movement as 8/10. The pain was limiting his daily activities and had not responded to a previous 5-day course of celecoxib. Concomitantly, the patient also referred asthenia and anorexia without significant weight loss. There was a medical history of hypertension controlled with lisinopril 20 mg daily. Physical examination showed the patient was haemodynamically stable, eupnoeic and afebrile. There was bilateral pitting oedema of the dorsum of the hands (Fig. 1). The patient also had swelling, tenderness and decreased range of motion of the joints of the hand, wrists, elbows, shoulders, knees and ankles. The remainder of the physical examination was unremarkable. 


\section{METHODS AND PROCEDURES}

Laboratory findings showed normocytic normochromic anaemia (haemoglobin $11 \mathrm{~g} / \mathrm{dl}$ ), an increased erythrocyte sedimentation rate (ESR $77 \mathrm{~mm} / \mathrm{h}$ ) and a raised C-reactive protein (CRP $9.12 \mathrm{~g} / \mathrm{dl}$ ). Serum autoantibodies (antimitochondrial, antinuclear, extractable nuclear antigen antibodies, antineutrophil cytoplasmic, anti-smooth muscle) and rheumatoid factor were negative. Urine and blood cultures were negative. Ultrasound and x-ray (Fig. 2) of the patient's hands demonstrated soft tissue swelling and extensor tenosynovitis without bone erosions.

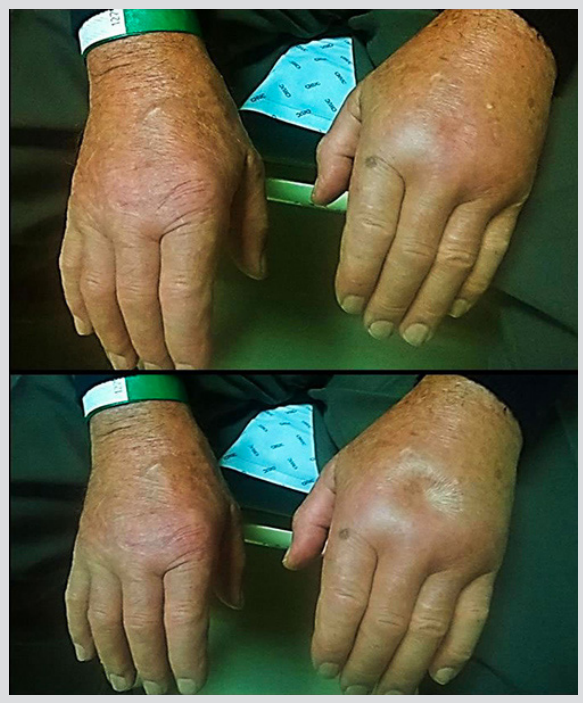

Figure 1. Soft tissue swelling and pitting oedema of the hands

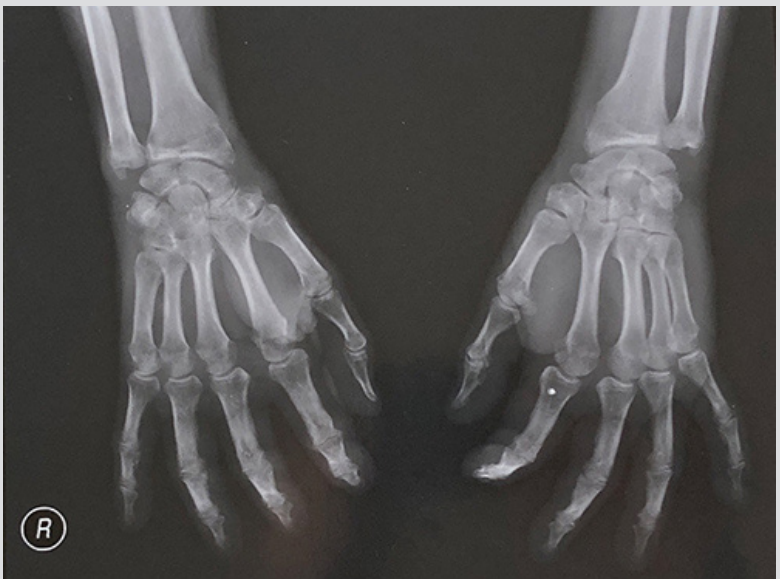

Figure 2. Plain $x$-ray of both hands

The clinical diagnosis of RS3PE was suggested and the patient was prescribed prednisolone $15 \mathrm{mg}$ daily. All previous signs and symptoms resolved after 1 week. Haemoglobin values remained at $11 \mathrm{~g} / \mathrm{dl}$, but the ESR and CRP normalized to $5 \mathrm{~mm} / \mathrm{h}$ and $0.27 \mathrm{mg} / \mathrm{dl}$, respectively. In order to exclude an underlying malignancy, a chest abdomen pelvis (CAP) computed tomography (CT) scan was performed and revealed a mass in the caecum measuring $29 \times 19 \times 32 \mathrm{~mm}$ (posteroanterior $\times$ transversal $\times$ longitudinal axis) and a contiguous $7 \mathrm{~mm}$ lymph node with high contrast enhancement, but no other anatomical changes (Fig. 3). Colonoscopy confirmed a vegetant mass in the caecum encompassing the ileocaecal valve. Biopsy of the mass revealed a low-grade adenocarcinoma. Serological levels of carcinoembryonic antigen were normal $(1.8 \mathrm{ng} / \mathrm{ml})$.

In September 2015 the patient underwent a laparoscopic right hemicolectomy (prednisolone was tapered until surgery). Histopathological examination of the surgical specimen confirmed a low-grade adenocarcinoma with extension to the muscularis propria and lymphovascular invasion. The resection margins were clear, and 12 mesocolic lymph nodes were excised without metastasis ( $p$ T2N0). Therefore, as this was an early stage cancer no further treatment was given and the patient did not resume corticotherapy.

After 3 years of follow-up there was no evidence of neoplastic relapse (Fig. 4) or RS3PE syndrome.

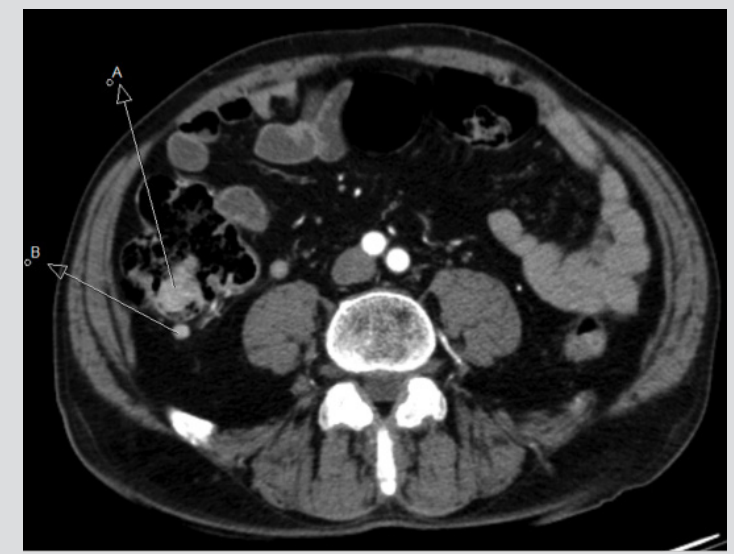

Figure 3. CAP-CT, August 2015: (A) tumour of the caecum; (B) lymph node

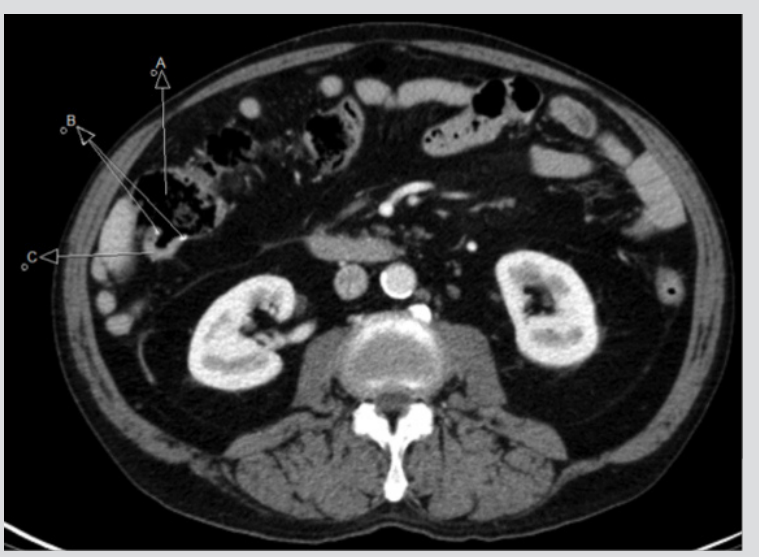

Figure 4. CAP-CT, September 2018: (A) colon; (B) metallic suture; (C) ileum 


\section{DISCUSSION}

Multiple subtypes of RS3PE have been described including autoimmune diseases, medication-induced syndrome as well as idiopathic forms. An association with haematological and solid tumours has also been reported, but in clinical practice such an occurrence is rare ${ }^{[1,2]}$. The mechanisms underlying RS3PE are not fully understood. The condition has been associated with elevated levels of vascular endothelial growth factor (VEGF) which explains the synovial inflammation and vascular permeability ${ }^{[3]}$.

The literature describes the presence of constitutional symptoms and diminished response to low-dose corticotherapy as specific characteristics related to paraneoplastic RS3PE syndrome ${ }^{[4]}$. In our patient, the systemic symptoms were present but the patient had a complete response to corticotherapy. The absence of RS3PE3 relapse after surgical extraction of the tumour despite the suspension of corticotherapy suggests the tumour played a major role in the aetiopathogenesis of the syndrome. A similar response to surgical resection of the tumour or to chemotherapy has been reported in other cases ${ }^{[5,6]}$.

In conclusion, this case report shows that acute clinical awareness is mandatory in treating elderly patients with RS3PE syndrome since this could be an opportunity to diagnose cancer at an early, curable stage. Further research should explore whether or not cancer screening should be systematically performed in these patients.

\section{REFERENCES}

1. McCarty DJ, O'Duffy JD, Pearson L, Hunter JB. Remitting seronegative symmetrical synovitis with pitting edema. RS3PE syndrome. JAMA 1985;254:2763-2767.

2. Li H, Altman RD, Yao Q. RS3PE: clinical and research development. Curr Rheumatol Rep 2015;17:49.

3. Arima K, Origuchi T, Tamai M, Iwanada N, Huang M, et al. RS3PE syndrome presenting as vascular endothelial growth factor associated disorder. Ann Rheum Dis 2005;64:16531655.

4. Tunc SE, Arslan C, Ayvacioglu NB, Sahin M, et al. Paraneoplastic remitting seronegative symmetrical synovitis with pitting edema (RS3PE syndrome): a report of two cases and review of the literature. Rheumatol Int 2004;24:234-237.

5. Vinci M, Malaguarnera L, Pistone G. RS3PE and ovarian cancer. Ann Rheum Dis 2001;60:429-430.

6. Sakamoto T, Ota S, Haruyama T, Ishihara M, Natsume M, Fukasawa Y, et al. A case of paraneoplastic remitting seronegative symmetrical synovitis with pitting edema syndrome improved by chemotherapy. Case Rep Oncol 2017;10:1131-1137. 